\title{
Neural Signal Processing: The Underestimated Contribution of Peripheral Human C-Fibers
}

\author{
Christian Weidner, ${ }^{1}$ Martin Schmelz, ${ }^{1}$ Roland Schmidt, ${ }^{2}$ Björn Hammarberg, ${ }^{2}$ Kristin Ørstavik, ${ }^{3}$ \\ Marita Hilliges, ${ }^{4}$ H. Erik Torebjörk, ${ }^{2}$ and Hermann O. Handwerker ${ }^{1}$ \\ ${ }^{1}$ Department of Physiology and Experimental Pathophysiology, University of Erlangen/Nürnberg, 91054 Erlangen, \\ Germany, ${ }^{2}$ Department of Clinical Neurophysiology, University of Uppsala, 75185 Uppsala, Sweden, ${ }^{3}$ Department of \\ Neurology, Ullevål Hospital, 0407 Oslo, Norway, and ${ }^{4}$ Department of Basic Oral Sciences, Karolinska Institutet, 14104 \\ Huddinge, Sweden
}

The microneurography technique was used to analyze usedependent frequency modulation of action potential (AP) trains in human nociceptive peripheral nerves. Fifty-one single C-afferent units (31 mechano-responsive, 20 mechanoinsensitive) were recorded from cutaneous fascicles of the peroneal nerve in awake human subjects. Trains of two and four suprathreshold electrical stimuli at interstimulus intervals of 20 and $50 \mathrm{msec}$ were applied to the receptive fields of single identified nociceptive units at varying repetition rates. The output frequency (interspike interval) recorded at knee level was compared with the input frequency (interstimulus interval) at different levels of accumulated neural accommodation.

At low levels of use-dependent accommodation (measured as conduction velocity slowing of the first action potential in a train), intervals between spikes increased during conduction along the nerve. At increasing levels of neural accommodation, intervals decreased because of a relative super- normal period (SNP) and asymptotically approached the minimum "entrainment" interval of the nerve fiber $(11 \pm 1.4$ $\mathrm{msec}$ ) corresponding to a maximum instantaneous discharge frequency (up to $190 \mathrm{~Hz}$ ).

For neural coding, this pattern of frequency decrease at low activity levels and frequency increase at high levels serves as a mechanism of peripheral contrast enhancement. The entrainment interval is a good minimum estimate for the duration of the refractory period of human $\mathrm{C}$-fibers.

At a given degree of neural accommodation, all afferent C-units exhibit a uniform pattern of aftereffects, independent of fiber class. The receptive class of a fiber only determines its susceptibility to accommodate. Thus, the time course of aftereffects and existence or absence of an SNP is fully explained by the amount of preexisting accommodation.

Key words: microneurography; C-fibers; neural coding; refractory period; accommodation; post-excitatory effects
During the time period immediately after an action potential (AP), at least four successive periods of post-excitatory conduction and excitability modulation are known, as outlined in Figure 1. The absolute refractory period (ARP) is directly followed by the relative refractory period (RRP), during which action potentials are conducted slower as would be expected, according to the Hodgkin and Huxley membrane theory (Hodgkin and Huxley, 1952). After the relative refractory period, a supernormal period (SNP) with increased conduction velocity (cv) and excitability may follow in some nerve fibers, but this is not always observed (see Fig. 1, solid and dotted line) (Lucas, 1917; Swadlow and Waxman, 1976; Raymond and Lettvin, 1978; Kocsis et al., 1979; Kiernan et al., 1997; Weidner et al., 2000a). After the shortlasting SNP, a period of conduction velocity slowing and reduced excitability can be regularly observed [hypoexcitable period (HP)]. This period has sometimes been subdivided into a shortlasting (H1) and a long-lasting (H2) subnormal period, both

\footnotetext{
Received Dec. 6, 2001; revised March 7, 2002; accepted April 11, 2002.

This work was supported by a Max-Planck price grant to H.E.T., Deutsche Forschungsgemeinschaft Grant SFB 353, Swedish Medical Research Council Project 5206, and a grant to R.S. from the Swedish Foundation for Brain Research. We are particularly grateful to Richard Carr for his valuable advice.

Correspondence should be addressed to Dr. Christian Weidner, Institut für Physiologie und Experimentelle Pathophysiologie, Universität Erlangen/Nürnberg, Universitätsstrasse 17, D-91054 Erlangen, Germany. E-mail: weidner@physiologie1.unierlangen.de.

Copyright (C) 2002 Society for Neuroscience $\quad 0270-6474 / 02 / 226704-09 \$ 15.00 / 0$
}

known to be additive for a number of conditioning action potentials (Bergmans, 1968; Shin and Raymond, 1991). The longlasting (H2) period has been examined extensively in many species and is known to be the reason for conduction velocity slowing on repetitive stimulation (Raymond and Lettvin, 1978; Raymond, 1979; Shin and Raymond, 1991). In humans (Serra et al., 1999; Weidner et al., 1999) and animals (Raymond et al., 1990; Thalhammer et al., 1994; Gee et al., 1996), it has been shown to correlate with the receptive properties of the nerve fibers. The H2 period, as a cumulative and prolonged (up to minutes) aftereffect, is an integral parameter of the overall level of axonal activity and serves as a kind of "nerve traffic indicator." During this period, it is conceivable that not only the electrical threshold and conduction velocity of a conditioned action potential are changed with the degree of neural activity, but also that the time course of its post-excitatory effects may be influenced, as has been shown for human A-fibers (Kiernan et al., 1997). This in turn may influence intraburst frequencies of conditioned groups of action potentials.

In humans, we reported recently that supernormal conduction, assessed as cv speeding, can be found in mechano-insensitive (MI) but not in mechano-responsive (MR) C-fibers when single conditioning APs are interposed in a regular train of APs (at 0.25 $\mathrm{Hz}$ ) (Weidner et al., 2000a). Supernormal conduction during the SNP was always relative, i.e., the conditioned AP was faster than the previous AP, but not faster than an AP after a long rest period (basal conduction velocity without HP). MI fibers are known for 
their pronounced activity-dependent cv slowing ( $6 \%$ for $\mathrm{MI}$, $0.6 \%$ for $\mathrm{MR}$ units at $1 / 4 \mathrm{~Hz}$ ), which also occurs during low repetition rates (Weidner et al., 1999).

The present study aims at investigating the effects of preexisting activity-dependent hypoexcitability and cv slowing [preexisting slowing (PS)] on the SNP. Furthermore, we were interested in the possible physiological impact of SNP on signal transduction and coding in human $\mathrm{C}$-fibers. According to our hypothesis, the sequence of SNP and HP acts as a contrast enhancement mechanism (Weidner et al., 2000a). An AP occurring during SNP will close up to the preceding AP, whereas one occurring later during the HP will increase its delay from the preceding AP. Here we studied whether such a contrast enhancement mechanism extends to trains of APs. We were further interested in knowing to what extent these phenomena are expressed in different $\mathrm{C}$-fiber classes at different levels of activity.

Part of this work has been published previously in abstract form (Weidner et al., 2000b).

\section{MATERIALS AND METHODS}

Subjects. In 23 healthy young subjects (12 male, 11 female, aged 19-34 years), microneurography was used to record 51 cutaneous afferent C-fibers from the peroneal nerve at knee level. All participants gave their informed written consent. This study was performed in our research laboratories in Uppsala and Erlangen according to the Declaration of Helsinki with previous approval by the local ethics committees.

Recording technique. The technique used to record single C-fibers from humans by means of microneurography has been published in detail by Torebjörk (1974) and is therefore only summarized briefly here. Weak electrical pulses applied through the uninsulated tip of a microelectrode of $0.2 \mathrm{~mm}$ diameter (microneurography active/2 M $\Omega$; Frederik Haer Inc., Bowdoinham, ME) guided its manual insertion into a cutaneous fascicle of the peroneal nerve dorsolateral to the fibular head. A reference microelectrode was placed subcutaneously nearby, and the recorded nerve signal was displayed and stored on a PC and also passed through an audio-amplifier. Final adjustment of the electrode position was guided by the characteristic sound of multifiber discharges evoked by gently stroking the skin in the innervation territory (lower leg or foot dorsum).

A pointed steel probe with a small contact surface (1 $\mathrm{mm}$ in diameter) was moved on the skin until the strong electrical search stimuli $(0.2 \mathrm{msec}$, $50 \mathrm{~mA}$ ) from an insulated constant current stimulator (Digitimer DS7, Digitimer, Hertfordshire, UK) evoked C-fiber action potentials with reproducible latency. The bias toward mechano-responsive $\mathrm{C}$-fibers was minimized because this stimulus strength is known to exceed even the high electrical thresholds of mechano-insensitive units (Weidner et al., 1999). To reduce the transcutaneous electrical resistance, two needle electrodes of $0.2 \mathrm{~mm}$ shaft diameter were inserted $5 \mathrm{~mm}$ apart in the innervation territory of an identified single $\mathrm{C}$-fiber for repetitive intracutaneous electrical stimulation (usually $1 / 4 \mathrm{~Hz}, 0.2 \mathrm{msec}, 80-150 \mathrm{~V}$, from an insulated Grass S 88 stimulator). The shortest distance between the stimulating needles in the skin and the recording electrode in the nerve was assessed in millimeters with a measuring tape. Room temperature was kept constant at $22-24^{\circ} \mathrm{C}$.

Characterization. C-units were characterized by the "marking" technique (Torebjörk and Hallin, 1974; Schmidt et al., 1995), i.e., any sudden increase and subsequent slow recovery of response latency was regarded as a sign of activation of the respective unit. Afferent $\mathrm{C}$-units were identified by their marking response to natural stimulation (i.e., mechanical or heat stimuli) of their innervation territories in the skin (Torebjörk, 1974). Mechanical stimuli were applied by calibrated von Frey filaments. The cutoff force for classification as mechano-responsive or mechano-insensitive nociceptors was set to $750 \mathrm{mN}$ as discussed elsewhere (Weidner et al., 1999). Heat stimuli (linear ramp $32-50^{\circ} \mathrm{C}$, $0.25^{\circ} \mathrm{C} \cdot \mathrm{sec}^{-1}$ ) were delivered from a halogen lamp feedback-controlled by a thermocouple attached to the skin (Beck et al., 1974), and the tolerance level of the subjects was the cutoff to determine heat sensitivity. The units were classified as mechano- and heat-responsive $(\mathrm{CMH})$, mechano-insensitive heat responsive $(\mathrm{CH})$, or mechano- and heatinsensitive $(\mathrm{CMiHi})$ afferents or sympathetic efferents. The two latter categories were distinguished by a marking response of the sympathetic units evoked by arousal stimuli such as unexpected shouting, mental arithmetic, or deep inspiration, all of which are known to elicit sympathetic reflexes in human skin nerves (Hallin and Torebjörk, 1970; Hallin and Torebjörk, 1974a,b; Hagbarth et al., 1972), and by the pronounced activity-dependent slowing of the $\mathrm{CMiHi}$ units known to clearly separate them from sympathetic units (Weidner et al., 1999).

Data acquisition and analysis. Signals from the recording electrodes were amplified and recorded on-line by a PC through an interface card (DAP, Microstar), using the SPIKE/SPIDI (Forster and Handwerker, 1990) and the Drever (Hammarberg et al., 2002) software package customized for this purpose. For the purpose of statistical testing, we used Statistica's (StatSoft, Tulsa OK) ANOVA. Where appropriate, nonparametric statistics were applied. Differences were regarded as significant at $p<0.05$. The appropriate corrections (Bonferroni) for repetitive testing were used.

Experimental protocol. After a rest period of at least $2 \mathrm{~min}$, pairs or quadruplets of suprathreshold electrical pulses at interstimulus intervals of 50 or $20 \mathrm{msec}$ were applied repetitively to the receptive field. Figure 2 shows a typical protocol for pair stimulation at an interstimulus interval of $50 \mathrm{msec}$, and Figure 5 shows a typical protocol for quadruplet stimulation at an interstimulus interval of $20 \mathrm{msec}$. The time between two pairs/quadruplets was stepwise decreased and in some of the experiments increased again, as indicated in Figures 2 and 5 (left panel). Steps were selected for individual units depending on the yielded effect of stimulation at the following fixed values: $16,8,6,4,2,1 \frac{1}{2}, 1,3 / 4$, and $2 / 3$ sec. The interval was decreased until it was either sufficient to evoke entrained APs (see Fig. 2, entrainment interval) (see below), or the concomitant activity-dependent threshold increase could no longer be compensated by increased stimulus strength without exceeding the tolerance limit of the subject. The limit of $666 \mathrm{msec}(2 / 3 \mathrm{sec})$ ensures that a new train never starts before the SNP of its precursor has vanished (Fig. 1).

The initial conduction velocity was calculated from the latency of the first action potential after rest. This latency was compared with the latency of the first AP in response to each stimulus train (pair or quadruplet), and the difference as a percentage of the initial latency was termed "preexisting slowing" (PS) (see Fig. 2) for that train. The preexisting slowing represents the cumulative long-lasting HP and therefore reflects neural accommodation, i.e., an integral measure for the nerve activity level.

An action potential after another at short latency will also be influenced by the short-lasting aftereffects of its precursor. The following analysis was used to quantify these effects. The latency of the second AP in a pair and for quadruplets also of the third and fourth AP in a train was assessed for each train, and their intervals (interspike interval; see Figs. 2, 5, distance between solid lines) were compared with the actual interstimulus interval of the stimulus train (see Figs. 2, 5, dotted lines). For pairs, the difference between interstimulus and interspike interval was termed $\Delta \mathrm{ISI}$ as indicated in Figure 2. If both action potentials in a pair have the same conduction velocity, their interspike interval should exactly match the interstimulus interval $(\Delta \mathrm{ISI}=0)$ as indicated in Figure 2 (dotted line). If the interspike interval was longer than the interstimulus interval (i.e., the second AP was slower than the first $=$ slowing), $\Delta$ ISI was defined as positive, whereas "speeding" of the second action potential leading to shorter interspike intervals was defined as negative (see Fig. 2).

Figure 2 shows that with increasing preexisting slowing of the first action potential, the interval to the second action potential $(\Delta \mathrm{ISI})$ decreases. The relation between $\Delta$ ISI and PS is illustrated as a "frequency modulation plot" in Figure 3. For statistical purposes, this representation of the data was summarized by three variables. First, a regression function was computed for the linear part of the frequency modulation plot (see Fig. 3, section 1 ) at low PS values. Note that only the increasing frequency steps were analyzed (see Fig. 3, top panel, full line), whereas the decreasing steps (see Fig. 3, top panel, dashed line) could not be completed for all experiments. This regression function is determined by two variables for each fiber. First, the slope of the regression line measures the degree of dependence between PS and $\Delta$ ISI. For example, a slope of $-4 \mathrm{msec} / \%$ means that an increase of PS by $1 \%$ decreases $\Delta$ ISI by $4 \mathrm{msec}$. Second, the intersection with the ordinate (i.e., the amount of $\Delta \mathrm{ISI}$ in the beginning of the experiment when PS $=0$ ) was called $\Delta \mathrm{ISI}_{\mathrm{PS}=0}$. A third variable measures the asymptotically approached entrainment interval (see Fig. 3, section 2), i.e., the stable interval between first and second AP at high PS (also indicated in Fig. 2). These three variables were taken for statistical comparison between fibers or fiber classes (see Fig. 4).

For quadruplets, PS and three $\Delta \mathrm{ISI}$ values $\left(\Delta \mathrm{ISI}_{1-2}, \Delta \mathrm{ISI}_{2-3}, \Delta \mathrm{ISI}_{3-4}\right)$ were assessed in an analogous way. Here, $\Delta \mathrm{ISI}$ is the difference between the real latencies of the second/third/fourth AP and those latencies that 


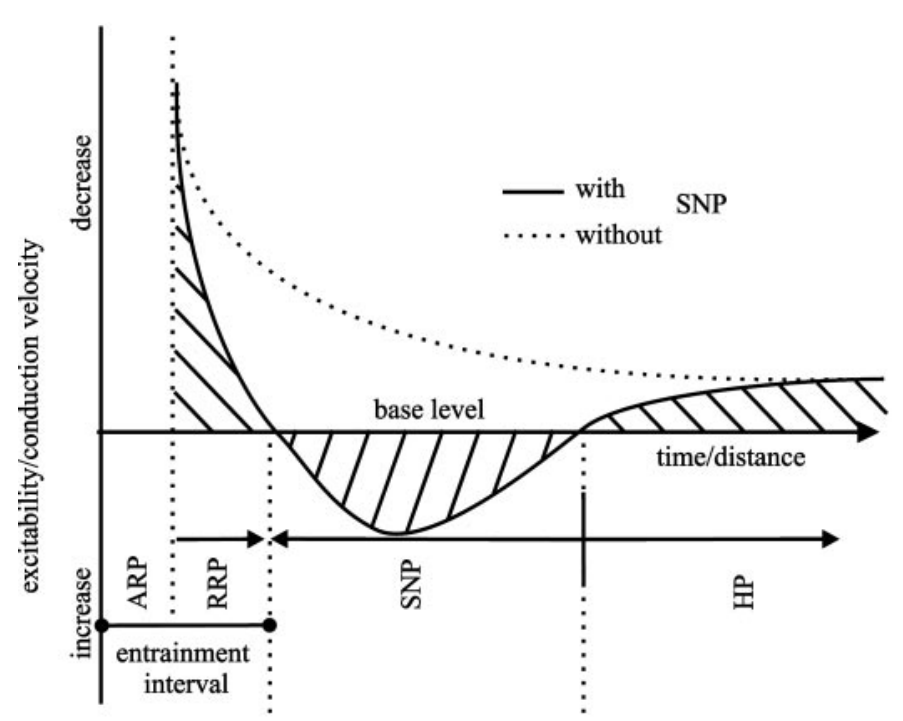

Figure 1. Model of postexcitatory effects: an action potential is followed by aftereffects with a certain time course or analogous spatial distribution on the axon (abscissa). An action potential is followed successively by (1) the absolute refractory period $(A R P)$, during which no second AP can be induced (frog A-fiber 1.5-2 msec); (2) the relative refractory period $(R R P)$ from end of APR to intersection of threshold with control level (frog A-fiber 1-2.5 msec; ARP + RRP human C-fiber, >5-10 msec); (3) the facultative supernormal period $(S N P)$, from end of RRP to second intersection with control level (frog A-fiber, $\sim 500 \mathrm{msec}$; human $\mathrm{A} \beta$-fiber, 15-20 msec; human A $\alpha$-fiber, $4 \mathrm{sec}$; human $\mathrm{C}$-fiber, $\sim 500 \mathrm{msec}$ ); (4) the hypoexcitable period $(H P)$ from end of SNP to asymptotic normalization of threshold (frog A-fiber and human C-fiber, minutes) [numbers taken from present work or Raymond and Lettvin (1978); Bostock and Bergmans (1994); Kiernan et al. (1997); Weidner et al. (2000a)]. The time course of postexcitatory conduction velocity or threshold change for a nerve fiber with an SNP is symbolized by the solid line. Arrows indicate whether the conditioned AP moves toward or away from the preceding conditioning AP. Given an infinitely long axon, a conditioned AP arising during RRP or SNP will be locked at the entrainment interval $\sim 1.7 \mathrm{msec}$ in rabbit central neurons (Kocsis et al., 1979) and $\sim 10 \mathrm{msec}$ in human C-fibers.

were calculated under the assumption that every AP follows its precursor with exactly the latency of the interstimulus interval, i.e., with the same conduction velocity (see Fig. 5 , dotted lines). Therefore $\Delta \mathrm{ISI}_{1-2}$ assesses the conditioning effect of the first AP in a train on the second in the same way as for pairs of stimuli. $\Delta \mathrm{ISI}_{2-3}$, however, relates the latency (conduction velocity) of the third AP to that of the second. Because the second AP is already influenced by the first, this influence is also included in the measure $\Delta \mathrm{ISI}_{2-3}$. For example, in the beginning of an experiment, at low PS, the aftereffects of the first AP slow down the cv of the second considerably (see Fig. 5, second AP $>20 \mathrm{msec}$ after first AP, i.e., to the right of the first dotted line). If this aftereffect persists until the third AP, the third AP should have the same cv as the second plus the cv changes induced by additional aftereffects of the second AP. Only this additional change in latency is measured by $\Delta \mathrm{ISI}_{2-3}$. $\Delta \mathrm{ISI}_{3-4}$ relates the latency of the fourth AP to that of the third in a comparable way. This is rationalized by the assumption that the conditioning effect of the first AP in a train outlasts the train duration and has to be considered effective for each of the following APs in the same trace. To summarize aftereffects of quadruplets, the modulation functions of single fiber recordings were averaged and a mean frequency modulation plot was assessed.

\section{RESULTS}

\section{Classification of units}

For the present study 51 human peroneal C-units were recorded by means of microneurography. According to their receptive properties, $25 \mathrm{C}$-afferents were classified as mechano-heatresponsive units (CMH units [conduction velocity (cv): $0.944 \pm$
$0.03 \mathrm{~m} \cdot \mathrm{sec}^{-1}$ ], 9 were classified as heat-responsive but mechanoinsensitive $\mathrm{CH}$ units $\left(\mathrm{cv}: 0.736 \pm 0.04 \mathrm{~m} \cdot \mathrm{sec}^{-1}\right)$, and 6 were classified as mechano-heat-insensitive CMiHi units (cv: $0.673 \pm$ $\left.0.04 \mathrm{~m} \cdot \mathrm{sec}^{-1}\right)$. Eleven units could not be tested for heat responsiveness because the recording position was lost. Six of them were mechano-responsive units of unknown heat class (CMix), cv: $\left.0.842 \pm 0.05 \mathrm{~m} \cdot \mathrm{sec}^{-1}\right)$, and five were classified as mechanoinsensitive of unknown heat class (CMix, cv: $0.795 \pm 0.08$ $\left.\mathrm{m} \cdot \mathrm{sec}^{-1}\right)$. For further evaluation, mechano-insensitive $(\mathrm{CH}$, $\mathrm{CmiHi}$, and CMix) and mechano-responsive ( $\mathrm{CMH}$ and $\mathrm{CMx})$ units were regarded separately, and subgroups (classes differing in heat sensitivity) were pooled together because conductive properties have been shown to be independent of heat sensitivity but to differ significantly between mechano-responsive and mechanoinsensitive units (Weidner et al., 1999). Mechano-responsive units had a significantly larger conduction velocity than the mechanoinsensitive units (MR $0.924 \pm 0.03 \mathrm{~m} \cdot \mathrm{sec}^{-1}, n=31$ vs MI $0.764 \pm 0.03 \mathrm{~m} \cdot \mathrm{sec}^{-1}, n=20$; ANOVA, $p=0.0003$; matched sample, $p=0.0003$; see next paragraph) as has been shown previously (Schmidt et al., 1995; Weidner et al., 1999).

The stimulation needles in the receptive fields were located at a mean recording distance of $243 \pm 11 \mathrm{~mm}$ for the 31 mechanoresponsive and $407 \pm 22 \mathrm{~mm}$ for the 20 mechano-insensitive units. Because this difference was statistically significant, the fact that the mechano-responsive units were more proximally situated (probably by chance) might have influenced the results. Therefore, a subpopulation of units was put together from 12 units of either class with matched conduction distance (ANOVA NS) and are referred to as the "matched sample."

\section{Trains of two}

Paired stimuli at 50 msec interstimulus intervals always induced a response pattern comparable to that shown in Figure 2.

Repetitive application of stimulus pairs initially provoked a pronounced increase of response latency, which then gradually stabilized at a new longer latency (preexisting slowing). Therefore, the rate of increase in PS slows down over time. Regardless of the rate of PS changes, the relation between PS and $\Delta$ ISI remains linear, as can be seen in the frequency modulation plot (Fig. 3). In our protocol, the repetition rate was in most cases increased again before the latency could stabilize completely. Therefore, we can only give minimum estimates for the amount of slowing induced by a certain frequency. A full quantitative analysis can be found in recent studies (Weidner et al., 1999, 2000a). This preexisting slowing differed significantly for mechano-insensitive as compared with mechano-responsive units. Table 1 summarizes amounts of preexisting slowing that could be reached by a given repetition rate for mechano-responsive and mechano-insensitive units.

At the beginning of a series of stimulus pairs, i.e., when no preexisting slowing of the first AP of a pair was present, $\Delta$ ISI was always positive (Fig. 2, slowing), i.e., the second AP was conducted slower than the first.

The amount of $\Delta$ ISI in the beginning of an experiment $\left(\Delta \mathrm{ISI}_{\mathrm{PS}=0}\right)$ was $11.0 \pm 1.56 \mathrm{msec}$ for MR units and $12.08 \pm 0.77$ msec for MI units. This difference between unit classes was not statistically significant (ANOVA NS/matched sample NS) (Fig. 4). An increase in repetition rate (neural activity level) led to an increase in PS and a decrease in $\Delta$ ISI. At a median repetition rate of $1 / 8 \mathrm{~Hz}(\mathrm{MI})$ or $1 / 2 \mathrm{~Hz}(\mathrm{MR}), \Delta \mathrm{ISI}$ became shorter than the interstimulus interval, indicating that the second AP was now faster than the first AP. At this repetition rate, mechano- 


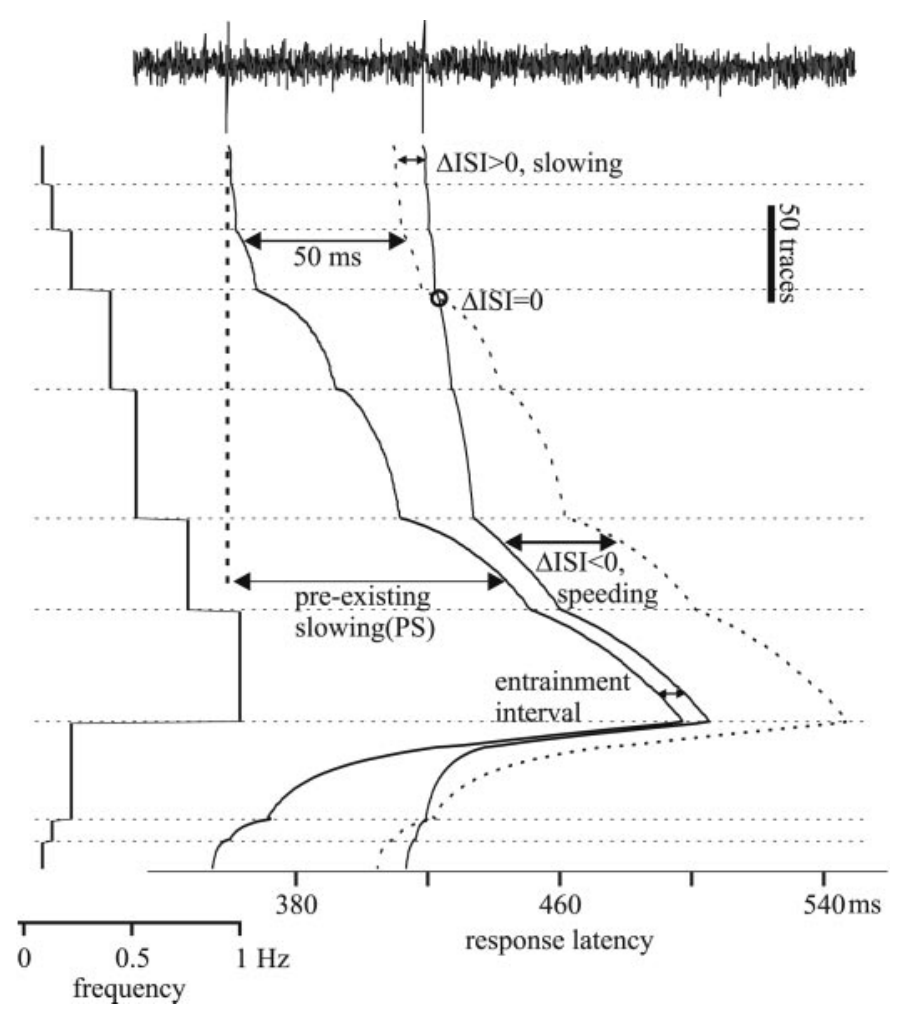

Figure 2. Discharge pattern of a $\mathrm{CMH}$ unit after pairs of stimuli at different repetition rates. Top, A trace of an original multifiber recording of the peroneal nerve in response to an electrical double pulse on the foot at a $50 \mathrm{msec}$ interspike interval is depicted. It shows a double response of a single human $\mathrm{C}$-fiber at the given response latency. This pair of stimuli is repeated at varying frequencies as indicated on the left panel (first increasing than decreasing steps). For each of the successive traces the response latencies of the pair of the action potentials are tracked by $t w o$ solid lines from top to bottom with a constant distance between two successive traces (i.e., the time scale of the ordinate is directly proportional to the stimulus frequency). Response latency increases for the first AP (pre-existing slowing) at higher levels of nerve activity. The second AP does not follow exactly $50 \mathrm{msec}$ (arrow labeled $50 \mathrm{~ms}$ ) later as indicated by the dotted line but appears even later at low levels of PS (slowing) or earlier at high levels (speeding). Speeding and slowing are summarized as $\Delta I S I$ (difference between interspike and interstimulus interval), and the point where $\Delta I S I=0$ (i.e., conduction velocities of the first and second action potential are equal) is marked. At a certain level of preexisting slowing, the two APs do not approach any closer and are considered to have reached the minimum entrainment interval.

insensitive units had a mean PS of $4.7 \pm 0.8 \%$ compared with $5.9 \pm 0.3 \%$ in MR units (ANOVA NS).

PS and $\Delta$ ISI are almost directly proportional for low values of PS. Therefore, for each fiber, a linear regression was computed for this linear part of the function (Fig. 3, section 1). On average, $r^{2}$ of this regression was $0.99 \pm 0.0008$. A second indicator for a functional relation between PS and $\Delta$ ISI is the smoothness of all function modulation plots (example in Fig. 3). This is most remarkable because underlying stimulus frequencies were changed stepwise, and the induced latency changes resembled those steps (visible steps in Fig. 2). In Figure 3, however, only the density of data points resembles the frequency steps.

At high repetition rates of pair stimulation (median $0.75 \mathrm{~Hz}$ for MI and 1.5 Hz for MR), a level of preexisting slowing ( $\sim 20-30 \%$ for MR and MI) could be reached sufficient to entrain the two action potentials (Fig. 2, section 2). On average, the interval between entrained APs (Figs. 2, 3, entrainment interval) was $8.3 \pm$

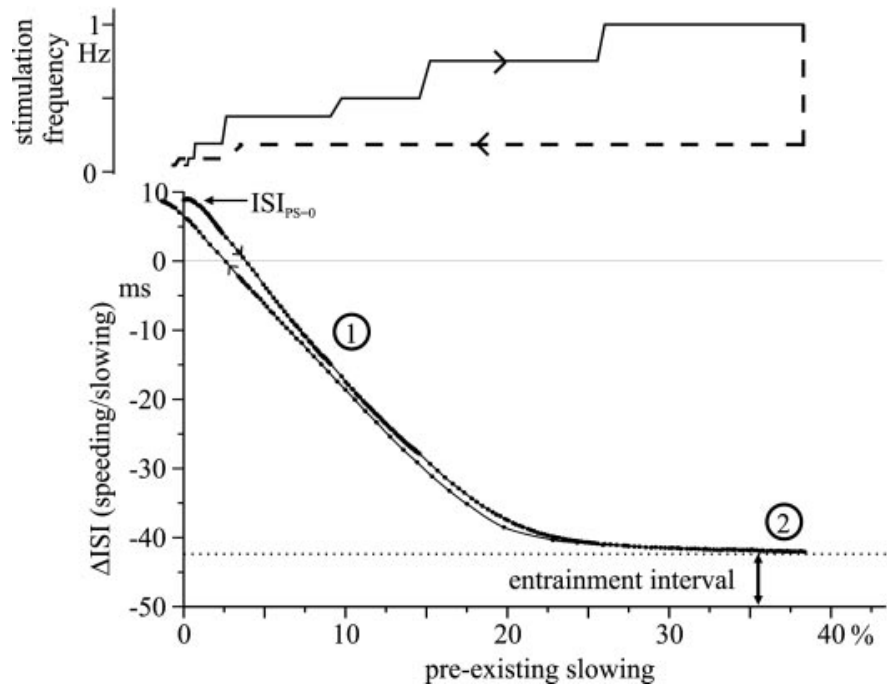

Figure 3. Frequency modulation plotted against preexisting slowing for the CMH unit of Figure 2. For the same recording as in Figure 2, the two variables of pre-existing slowing and $\Delta I S I$ (difference between interspikeand interstimulus interval) are plotted against each other, where $\Delta$ ISI represents slowing (positive) or speeding (negative). The top panel indicates the increasing (solid line) and decreasing (dashed line) stimulus frequency steps (ordinate) that were used to induce the preexisting slowing (abscissa). The bottom panel shows all single data points connected by a line with arrows indicating the increasing and decreasing frequency steps (hysteresis!). Note that after each frequency increase, PS changes in big steps (low density of data points), whereas a high density of data points indicates the asymptotic approach toward a new stable level of PS. The slope or smoothness of the function is not influenced by the data point density. Two different parts of the function can be separated. Section 1 marks the approximately linear segment in which $\Delta$ ISI decreases with increasing preexisting slowing; section 2 marks the asymptotic approach toward the entrainment interval, which is the duration between the dotted entrainment level (at $-42.03 \mathrm{msec}$ ) and the abscissa at $-50 \mathrm{msec}$.

$0.7 \mathrm{msec}(120 \mathrm{~Hz})$ for MR units and $10.4 \pm 1.2 \mathrm{msec}(96 \mathrm{~Hz})$ for MI units.

To reveal possible differences of the frequency modulation functions between the two fiber classes, we compared the following three determining variables: the slope of the regression line, its intersection with the ordinate $\left(\Delta \mathrm{ISI}_{\mathrm{PS}=0}\right)$, and the entrainment interval. None of these three variables shown in Figure 4 exhibited a significant difference between the two unit classes (ANOVA, all NS/matched sample, all NS).

Therefore, all human $\mathrm{C}$-fibers at a given level of preexisting slowing show the same time course of short-term aftereffects. In this context, it should be noted again that the same degree of preexisting slowing is achieved at much lower activity levels by mechano-insensitive as compared with mechano-responsive units (Table 1) (Weidner et al., 1999).

\section{Trains of four}

In Figure 5, a typical response pattern after quadruplets of stimuli at interstimulus intervals of $20 \mathrm{msec}$ is shown.

For interstimulus intervals of $50 \mathrm{msec}$, the response pattern was comparable to the $20 \mathrm{msec}$ pattern, and no example is included. Figure 5 shows that all three conditioned action potentials in a quadruplet followed the same pattern of activity-dependent latency shift. At low-repetition rates, each AP was slower than its predecessor. On increasing levels of activation (PS) this cv difference between two neighboring APs decreased until reaching points of identical cv. These points are marked in Figure 5 as 


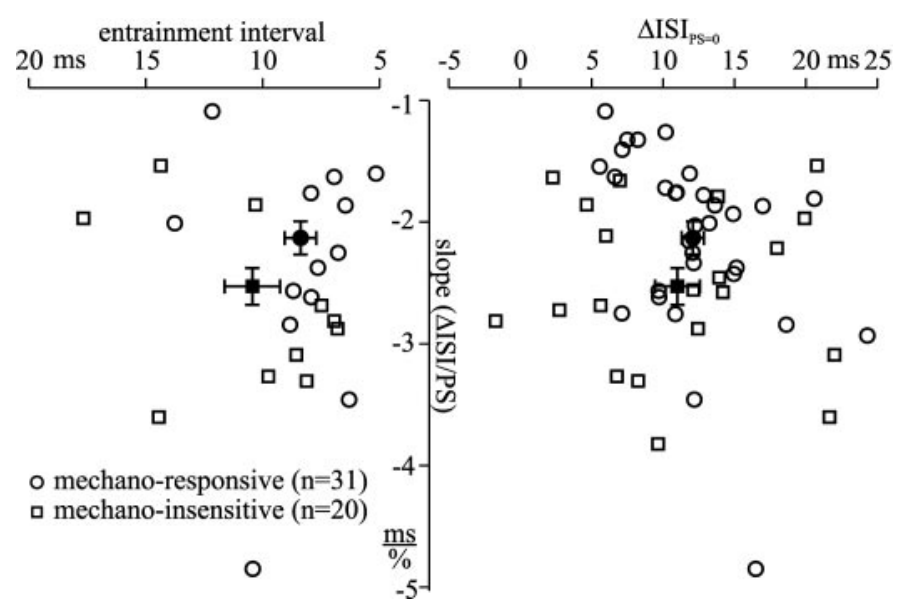

Figure 4. Frequency modulation functions of peripheral C-fibers summarized separately for mechano-insensitive and mechano-responsive units. This figure summarizes the three variables determined from the frequency modulation plots ( $\Delta$ ISI vs preexisting slowing at $50 \mathrm{msec}$ interstimulus interval) of all recorded fibers: two variables determine the linear regression function for section 1 , and the third determines the entrainment interval for section 2 in Figure 3. For the linear fit in section 1 , the intersection of the regression line with the ordinate $\left(\Delta \mathrm{ISI}_{\mathrm{PS}=0}\right.$; the interpolated $\Delta$ ISI when preexisting slowing $=0$ ) and the slope of the regression line are plotted against each other in the right panel of this figure. The left panel shows the entrainment interval plotted against $\Delta \mathrm{ISI}_{\mathrm{PS}=0}$. None of these three measures were significantly different for mechano-insensitive as compared with mechano-responsive units.

$\Delta \mathrm{ISI}_{1-2=0}, \Delta \mathrm{ISI}_{2-3=0}$, and $\Delta \mathrm{ISI}_{3-4=0}$, i.e., when the first (second, third) and the second (third, fourth) AP had the same cv. For further increases in neural activity, the cv ratio of neighboring APs reversed. Successively, the second (third, fourth) AP became faster than the first (second, third) AP, and their relative interval in the axon decreased as they propagated centrally. This activitydependent pattern of output frequency decrease followed by increase could be observed in successive order for all three pairs of neighboring APs and is depicted as a frequency modulation plot analogous to Figure 3 in Figure 6.

The insets show the average frequency modulation plots and confirm the consistency of this pattern in all nine units examined. The displayed range was limited by values of preexisting slowing that could be reached for all units. When the compared pair of APs was later in the train, the frequency modulation function was shifted to higher preexisting slowing. Hence, the PS at which two neighboring APs had the same cv $(\Delta \mathrm{ISI}=0)$ was on average $4.8 \%$ for $\Delta \mathrm{ISI}_{1-2}, 8.8 \%$ for $\Delta \mathrm{ISI}_{2-3}$, and $12 \%$ for $\Delta \mathrm{ISI}_{3-4}$ at an interstimulus interval of $20 \mathrm{msec}$. At an interstimulus interval of 50 msec, only the first two spikes in a train reached the same conduction velocity $\left(\Delta \mathrm{ISI}_{1-2}=0\right)$ at an average PS of $4.9 \%$. The single unit recordings in Figures 5 and 6 show that high levels of neural activity may affect the conduction velocity of all four APs such that their interspike intervals asymptotically approach the entrainment interval. The resulting output frequency might reach up to $100 \mathrm{~Hz}$ (entrainment interval $\sim 10 \mathrm{msec}$ ), although the input frequency was only 50 or $20 \mathrm{~Hz}$ (interstimulus interval 20 or 50 msec).

\section{DISCUSSION}

The aim of this study was a comprehensive analysis of postexcitatory effects of action potentials in different classes of human afferent $\mathrm{C}$-fibers as a function of their activity level. Our main finding is that short-lasting aftereffects following each action potential are dependent on the ongoing rate of spike activity. A particular activity level inducing a certain amount of activitydependent slowing fully determines the corresponding time course of post-excitatory effects regardless of the class of nociceptor. The sensory modality of the C-fibers (mechanoresponsive vs mechano-insensitive) only determines the amount of activity-dependent slowing induced by a certain level of ongoing activity. The relative supernormal period might allow for intermittent peak frequencies of up to $190 \mathrm{~Hz}$ (Fig. 4) (mean $113.2 \pm 2.6)$. It can only be observed in accommodated axons.

The modulation of conduction velocity studied here, as a parameter of post-excitatory effects in human C-fibers, can be regarded as equivalent to the more commonly studied threshold modulation, which is difficult to assess using microneurography in awake subjects (Raymond and Lettvin, 1978; Shin et al., 1994). The results presented here and in recent studies (Weidner et al., 1999, 2000a) are consistent with findings from threshold tracking in rat C-fibers (Shin and Raymond, 1991) and frog A-fibers (Raymond, 1979). Unlike threshold tracking experiments, the assessment of activity-dependent conduction velocity slowing is critically dependant on the point of AP generation. If this point moves proximally during an experiment, the conduction latency would decrease and vice versa. In animal experiments, it is indeed possible to yield a gradual latency decrease with increasing stimulus intensity. This can be explained by current spread, i.e., the nerve fiber can be excited further proximal with increasing current. Astonishingly, signs of current spread, especially the gradual latency decrease, are not observed in human skin, in which stimulus intensity is limited by the tolerance level of the subjects. Likewise, we found cutaneous receptive fields with low-threshold spots (10 mA) just $1 \mathrm{~mm}$ away from spots where even $100 \mathrm{~mA}$ could not excite the nerve fiber (Schmidt et al., 1998). Therefore the assessed latency changes can most likely be attributed to neural accommodation rather than current spread. Thus, modulation of interspike interval is a valuable method to assess postexcitatory effects as already described for compound action potentials in human A-fibers (Stys and Ashby, 1990).

Post-excitatory effects are composed of a number of distinct mechanisms, each having its own time course. We discuss the contributing phenomena in the following sections, always aware that their respective time courses overlap and that a single mechanism never fully explains the observed effect at a given time.

\section{The refractory periods of human C-fibers}

The ionic mechanisms for only two of the aftereffects after an AP are well known, for the absolute and relative refractory periods. Both refractory periods are explained by the time course of sodium and potassium currents as described in the HodgkinHuxley-Katz (HHK) model (Hodgkin and Huxley, 1952). High sodium inactivation $(h)$ and increased potassium conductance $\left(g_{\mathrm{K}}\right)$ as calculated from the HHK model make the axon leaky to current and reduce the probability of propagated depolarization. To our knowledge, the time course, the activity dependence, and the duration of the refractory period of human C-fibers have not been adequately explained hitherto. The time course of the early RRP and the end of the ARP cannot be assessed in microneurography studies because the subject would not tolerate stimuli exceeding the high thresholds during the early RRP. The late RRP that was studied here was found to be highly activity dependent. However, during the late RRP, the membrane potential is probably already strongly influenced by ionic mechanisms un- 


\section{Table 1. Preexisting slowing in both afferent fiber classes}

\begin{tabular}{|c|c|c|c|c|c|}
\hline Repetition rate & $1 / 16^{*}$ & $1 / 8^{*}$ & $1 / 4^{*}$ & $1 / 2^{*}$ & $1 / 1.5$ \\
\hline PS (mechano-insensitive) & $2.6 \%$ & $6 \%$ & $11.1 \%$ & $20.2 \%$ & $24.1 \%$ \\
\hline PS (mechano-responsive) & $0.7 \%$ & $1.6 \%$ & $4.3 \%$ & $11.4 \%$ & $18.5 \%$ \\
\hline
\end{tabular}

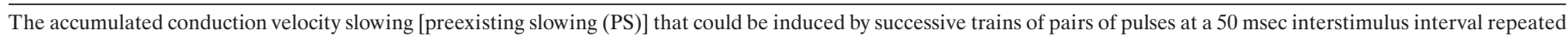

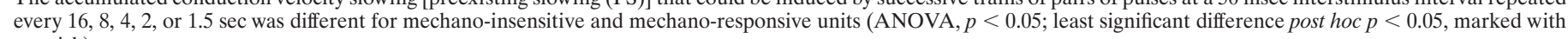
asterisk).

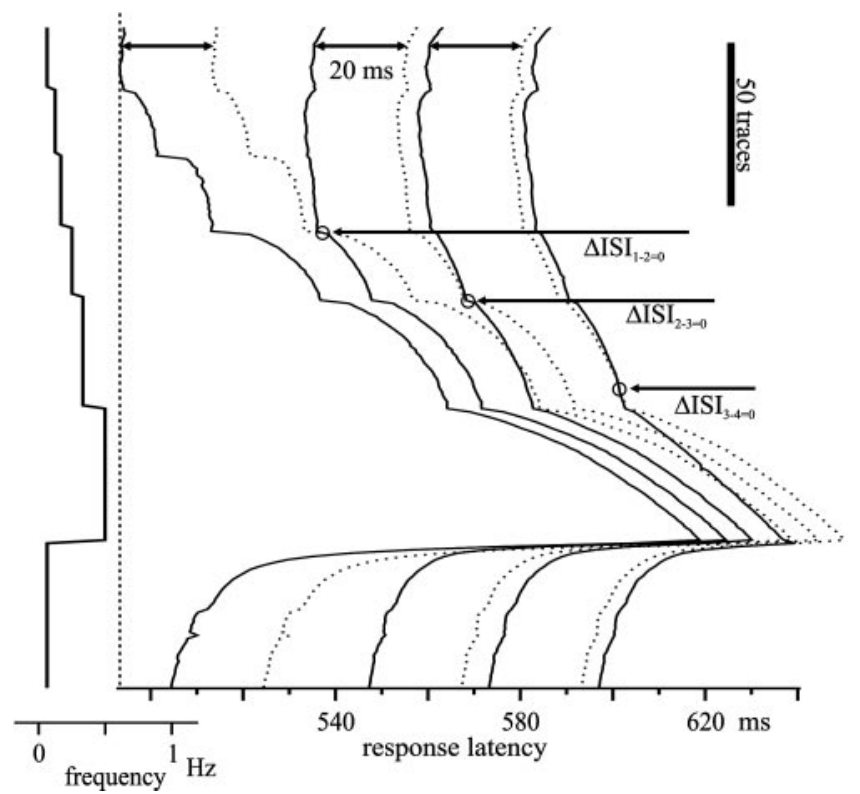

Figure 5. Response of a $\mathrm{CMH}$ unit to quadruplets of stimuli at an interstimulus interval of $20 \mathrm{msec}$. Latencies of successive responses to quadruplets of stimuli, each at an interval of $20 \mathrm{msec}$, are depicted (analogous to Fig. 2) from top to bottom in successive order at their individual latencies (abscissa). The dotted lines indicate the latency at an interval of exactly $20 \mathrm{msec}$ from the previous action potential for each of the last three responses in each trace. The points where the conduction velocity of an action potential was equal to that of the previous AP in the same quadruplet are marked $\left(\Delta I S I_{1-2}, \Delta I S I_{2-3}, \Delta I S I_{3-4=0}\right)$.

derlying the arising SNP, which has a pronounced activity dependence as shown in the present study.

By definition, the relative refractory period ends when the threshold or the conduction velocity has reached the control level again. This definition, however, is only sensible if the RRP is followed by an SNP (i.e., crosses and goes under the control level). Alternatively, if the RRP is immediately followed by a long-term depression, the control level may only be reached after several minutes (Fig. 1 solid line vs dotted line or Fig. 7 foreground vs background). The duration of the relative refractory period, as determined in the present study, is not identical to that derived from the HHK model because this model does not include supernormality and late aftereffects. In human C-fibers, the SNP is only present in conditioned axons, and therefore the RRP is not well defined in an unconditioned $\mathrm{C}$-fiber axon. In conditioned $\mathrm{C}$-fibers at maximum neural activity levels (i.e., maximum SNP), the entrainment interval was on the order of $10 \mathrm{msec}$ and can serve as a minimum estimate for the duration of the RP. To our knowledge, no other study contributes an estimate for the duration of the RP in human C-fibers. When we compare our results with data from animals (Grundfest and Gasser, 1938; Shin and Ray-
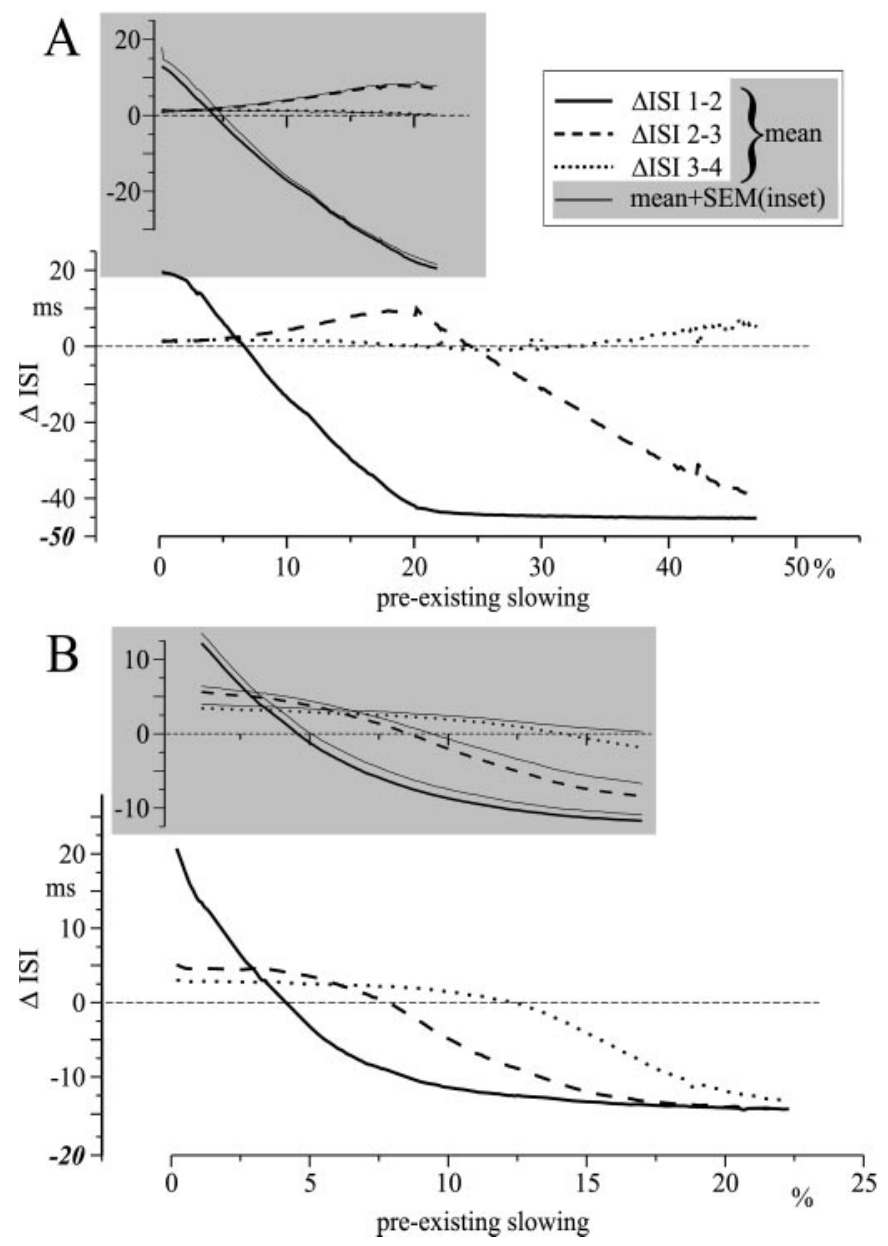

Figure 6. Frequency modulation function for quadruplets at 20 and 50 msec interstimulus interval (example and mean + SEM). $\Delta$ ISI is plotted against PS (analogous to Fig. 3) for each of the three conditioned APs $\left(\Delta \mathrm{ISI}_{1-2}, \Delta \mathrm{ISI}_{2-3}, \Delta \mathrm{ISI}_{3-4}\right.$, as indicated in Fig. 5). Here, $\Delta \mathrm{ISI}$ is the difference of the real latency of a conditioned AP to the assumed latency if it had the same conduction velocity as the preceding AP (i.e., assuming that each AP would occur exactly at the interstimulus interval of 20 or 50 msec after its precursor). $A$ shows the three frequency modulation functions for a single $\mathrm{CMH}$ fiber. The gray inset (same abscissa) shows the average frequency modulation function (mean/mean + SEM) for five $\mathrm{C}$-fibers at an interstimulus interval of $50 \mathrm{msec}$. For four $\mathrm{C}$-fibers, frequency modulation functions in response to quadruplets at an interstimulus interval of $20 \mathrm{msec}$ are depicted in the same way in $B$ (example unit identical to Fig. 5).

mond, 1991), it seems that the duration assessed here is quite long despite it being only a minimum estimate.

\section{The supernormal period}

The supernormal period after the RRP is known for A- and C-fibers in different animal models (Raymond and Lettvin, 1978; Raymond, 1979; Carley and Raymond, 1987; Shin and Raymond, 


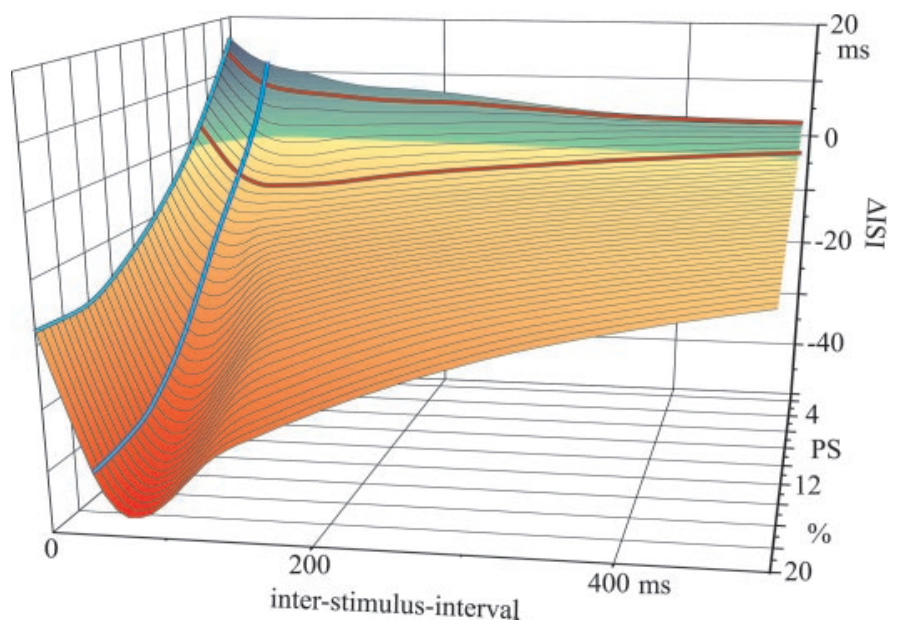

Figure 7. Presumed time course of postexcitatory effects in human $\mathrm{C}$-fibers. This three-dimensional figure summarizes the effects of preexisiting slowing (PS, \% decrease of conduction velocity) and interstimulus interval on $\triangle$ ISI (supernormality in yellow-red and subnormality in green). Supernormality of conduction is most pronounced at intense PS and short interstimulus intervals, whereas subnormality is found at a low degree of PS. Blue lines represent findings from the present study (frequency modulation function at 20 and $50 \mathrm{msec}$ interstimulus interval). Data for the red lines is taken from previous work (Weidner et al., 2000a).

1991; Bostock and Bergmans, 1994; Kiernan et al., 1997). It has been speculated to be caused by membrane depolarization induced by extracellular potassium accumulation (Gilliatt and Willison, 1963; Swadlow and Waxman, 1976; Kocsis et al., 1979; Kocsis et al., 1983; Bowe et al., 1987). However, our results strongly support another hypothesis (Barrett and Barrett, 1982) attributing the afterdepolarization to a mere discharge of the capacity of the axonal membrane at a time when all channels involved in the depolarizatiion and repolarization of the AP are closed again. Recording intracellularly from the myelinated axons of lizard and frog, Barrett and Barrett (1982) found a long-lasting (half-life time 20-100 msec) afterdepolarization after an AP only if the resting membrane potential was hyperpolarized (more negative than -60 to $-45 \mathrm{mV}$, dependent on species). Its magnitude linearly increased with increasing preexisting hyperpolarization. Likewise, the present study shows that in human C-fibers, the SNP is dependent on the present degree of neural accommodation. In terms of conduction velocity this means that the presence of an activity-induced slowing of conduction is the prerequisite for conduction velocity speeding in the SNP, and its degree determines the amount of speeding. Figure 3 shows that this increase of supernormality with increasing neural accommodation (PS) is approximately linear at lower levels of accommodation. This means that at a given time after an accommodated action potential (here 20 and $50 \mathrm{msec}$ ), the SNP diminishes the preexisting slowing by a constant percentage. We never observed a complete reversal of preexisting slowing; i.e., even in the supernormal phase, conduction velocity never exceeded the initial unconditioned velocity.

The passive capacitative current shown to be responsible for the afterdepolarization in myelinated axons can likewise explain the SNP in human C-fibers. Given a strong voltage dependence of the repolarizing $\mathrm{K}$ channels, the membrane potential would not be expected to completely repolarize after each AP, because the $\mathrm{K}$ channels would have already closed at a moderately depolarized membrane potential a brief time after the AP. If the trans- membrane resistance is high, a depolarization that outlasts the closure of the $\mathrm{K}$ channels would be terminated only with a long time constant, and this may explain an outlasting SNP. In human $\mathrm{C}$-fibers, the voltage dependence of the repolarizing $\mathrm{K}$ channels therefore seems to be just adequate to fully repolarize the membrane after an AP starting from the resting membrane potential. If an AP starts from a hyperpolarized membrane potential, it can rapidly repolarize only to a level around the original resting membrane potential, and considerably more time is required to reach the hyperpolarized level again, which then appears as relative supernormality.

Both major C-fiber classes (mechano-responsive and mechanoinsensitive) have exhibited an identical time course of the SNP and the same dependence on hyperpolarization. This leads to the assumption that both the repolarizing $\mathrm{K}$ current and the time constant of the membrane are independent of fiber class. Note that the time constant is independent of fiber diameter $(\tau=$ $R_{\text {Membrane }} \times C_{\text {Membrane }}, R_{\text {Membrane }} \sim 1 / r, C_{\text {Membrane }} \sim r$, i.e., $\tau=$ constant), allowing for differences of geometry.

\section{The entrainment interval}

At higher levels of neural activity (i.e., pronounced PS), the SNP asymptotically approaches the entrainment interval. This finding reflects the fact that the capability of the SNP to speed up a succeeding AP is limited by the refractory period of its predecessor. Therefore, the inverse of the entrainment interval determines the maximum discharge frequency of $\mathrm{C}$-fibers. This maximum frequency can reach $190 \mathrm{~Hz}$, corresponding to an entrainment interval of $5.3 \mathrm{msec}$. Of course this maximum frequency can only be reached in an accommodated nerve. The neural activity level must slow down conduction by $>20 \%$ to allow sufficient relative speeding for entrained AP trains at $100 \mathrm{~Hz}$. As shown in Figure 5 , this also holds true for longer trains (four APs) in which an increase in neural accommodation (PS) allows an increasing number of APs to be entrained. Therefore, the present study allows us to determine the maximum discharge frequency of human C-fibers within spike trains (observed range 57-194 Hz).

\section{The late hypoexcitable period}

The late hypoexcitable period follows the refractory period either directly (in an unconditioned axon) or after the supernormal period (in an accommodated axon). Its ionic basis is most likely an increased activity of the electrogenic $\mathrm{Na} / \mathrm{K}$ pump, which induces hyperpolarization and is blocked by ouabain (Rang and Ritchie, 1968; Raymond and Lettvin, 1978). In this study, we assessed the present state of subnormality by assessing the degree of conduction velocity slowing of the first action potential in a train, assuming that this level of subnormality underlies the full train and is only modified by subsequent short-lasting aftereffects. This approach is rationalized by the steady slowing of the conduction speed of the first AP in a train during repetitive stimulation. In the present study, we could show that the accumulated long-lasting subnormality provides the margin for possible speeding of succeeding APs and constitutes the basis of its modality dependence.

\section{Summary of aftereffects}

Taking results from a previous study (Weidner et al., 2000a) and the present results together we can give a comprehensive description of aftereffects in human C-fibers shown in Figure 7. The conditioning effect of an action potential on its successor $(\Delta \mathrm{ISI})$ depends on the degree of neural accommodation (present results) and on the interstimulus interval of conditioning and conditioned 
AP (previous study). In the present study we assessed the relation between $\Delta$ ISI and PS for two fixed interstimulus intervals of 50 and $20 \mathrm{msec}$ (Fig. 7, blue lines). In the previous study, $\Delta$ ISI (called latency shift in that study) was assessed independent of the interstimulus interval for two stable values of PS (0.6 and 6\%) (Fig. 7, red lines). In Figure 7 these data points are embedded in a three-dimensional grid of predicted afterpotentials.

\section{Modality dependence}

In a previous study we were able to show that activity-dependent slowing in human C-fibers is modality dependent to a degree that even allows unambiguous classification of units based only on this measure (Weidner et al., 1999). In a study describing the SNP of human C-fibers for the first time (Weidner et al., 2000a), we speculated that the observed activity dependence of the SNP might be related to the activity dependence of the subnormality. The present study clearly shows that preexisting slowing is a prerequisite for the SNP. The described modality dependence of the SNP should therefore be regarded as a consequence of the modality dependence of the subnormal period. The membrane mechanism inducing the cumulative activity-dependent slowing seems to be responsible for all the observed differences of action potential propagation in the two afferent unit classes. It could therefore be a promising pharmacological target to selectively impair conduction of mechano-insensitive fibers.

\section{Peripheral signal processing}

In the present study we have shown that a train of impulses reaches the knee at a lower rate than the stimulus frequency, with which they were generated in the receptive field of the unit, when the neural activity level is low. In contrast, high neural background activity could increase intra-train frequency from 20 or 50 $\mathrm{Hz}$ to the entrained maximum of the unit of $160 \mathrm{~Hz}$ in a train of four (Fig. 5) or $190 \mathrm{~Hz}$ in a train of two (Fig. 2). This pattern may act functionally as a contrast enhancement mechanism. It might very well account for part of the psychophysical windup phenomena seen with repetitive electrical stimulation, which is usually attributed to postsynaptic processing in the spinal cord. The temporal pattern of the spinal input is important for the magnitude of the perceived sensation, i.e., pain, as shown in previous experiments with intraneural microstimulation (Torebjörk et al., 1984; Jorum et al., 1989). Under pathophysiological conditions (e.g., during inflammation) when C-fibers are probably spontaneously active, this contrast enhancement can become meaningful for perception because two prerequisites are satisfied. First, entrainment of multiple action potentials could only be observed with sufficiently high intraburst frequencies (Fig. 6, compare $A$, $B)$. The typical response pattern for $\mathrm{C}$-fibers stimulated with inflammatory mediators consists of repetitive bursts at rather high intraburst frequencies. Second, sufficient neural accommodation, measured as preexisting slowing in this study, is the prerequisite for this contrast enhancement. Both unit classes develop the same aftereffects and thereby the same frequency modulation at a given degree of PS as summarized in Figure 4. However, the mechanoinsensitive fibers are more likely to reach high amounts of PS at modest stimulus frequencies as published recently (Weidner et al., 1999) and summarized for the present fiber sample in Table 1. Given an average slope of $2.5 \mathrm{msec} / \%$, an increase of preexisting slowing of $10 \%$ would be enough to increase discharge frequency from 20 to $40 \mathrm{~Hz}$ as the impulses traveled from the foot to the knee. For a preexisting slowing of $10 \%$, ongoing activity of $<1 / 4$ $\mathrm{Hz}$ in mechano-insensitive and $1 / 2 \mathrm{~Hz}$ in mechano-responsive fibers is required (pair repetition rate; Table 1). Even the median frequencies needed for entrainment [pair repetition rate of 0.75 $\mathrm{Hz}(\mathrm{MI})$ and $1.5 \mathrm{~Hz}(\mathrm{MR})]$ can be readily achieved by chemical stimulation (Schmelz et al., 2000). In our experiments, we often failed to reach the entrainment interval because of a concomitant (electrical) threshold increase above the tolerance level of the subject. This artificial limitation, however, is not present for the more physiological stimulation evoked with inflammatory mediators. During ongoing responses, for example those to capsaicin, additional electrical stimulation is often without effect, although the fiber can still be excited chemically. In other words, chemically induced activity in $\mathrm{C}$-nociceptors easily provokes preexisting slowing. Therefore contrast enhancement in peripheral nerve fibers seems to play a physiological role in both fiber classes, with predominance for mechano-insensitive fibers.

In conclusion, this is the first study to provide the maximum discharge rate of human $\mathrm{C}$-fibers and its inverse, the entrainment interval. The entrainment interval is also a minimum estimate for the duration of the refractory period. Furthermore, we could demonstrate that all human $\mathrm{C}$-fibers share a common time course of aftereffects as depicted in Figure 7. This time course is only dependent on the neural activity level and the activity-dependent slowing induced thereby. The absolute amount of neural activity necessary to induce a certain level of slowing is dependent on the class of C-fibers. Because mechano-insensitive $\mathrm{C}$-fibers show the same amount of slowing at lower activity levels, they are probably more effective at central synapses at moderate impulse activity levels.

We clearly demonstrate here that the C-fibers in human nerves enhance frequency contrasts of propagated information to an unexpectedly high degree of approximately one decade.

\section{REFERENCES}

Barrett EF, Barrett JN (1982) Intracellular recording from vertebrate myelinated axons: mechanism of the depolarizing afterpotential. J Physiol (Lond) 323:117-144.

Beck PW, Handwerker HO, Zimmermann M (1974) Nervous outflow from the cat's foot during noxious radiant heat stimulation. Brain Res 67:373-386.

Bergmans J (1968) Active and passive mechanisms in the recovery of single human motor axons from activity. Arch Int Physiol Biochim 76:135-138.

Bostock H, Bergmans J (1994) Post-tetanic excitability changes and ectopic discharges in a human motor axon. Brain 117:913-928.

Bowe CM, Kocsis JD, Waxman SG (1987) The association of the supernormal period and the depolarizing afterpotential in myelinated frog and rat sciatic nerve. J Neurosci 21:585-593.

Carley LR, Raymond SA (1987) Comparison of the after-effects of impulse conduction on threshold at nodes of Ranvier along single frog sciatic axons. J Physiol (Lond) 386:503-527.

Forster C, Handwerker HO (1990) Automatic classification and analysis of microneurographic spike data using a PC/AT. J Neurosci Methods 31:109-118

Gee MD, Lynn B, Cotsell B (1996) Activity-dependent slowing of conduction velocity provides a method for identifying different functional classes of $C$-fiber in the rat saphenous nerve. Neuroscience 73:667-675.

Gilliatt RW, Willison RG (1963) The refractory and supernormal periods of the human median nerve. J Neurol Neurosurg Psychiatry 26:136-143.

Grundfest H, Gasser HS (1938) Properties of mammalian nerve fibers of slowest conduction. Am J Physiol 123:307-318.

Hagbarth KE, Hallin RG, Hongell A, Torebjörk HE, Wallin BG (1972) General characteristics of sympathetic activity in human skin nerves. Acta Physiol Scand 84:164-176.

Hallin RG, Torebjörk HE (1970) Afferent and efferent C units recorded from human skin nerves in situ. A preliminary report. Acta Soc Med Ups 75:277-281.

Hallin RG, Torebjörk HE (1974a) Single unit sympathetic activity in human skin nerves during rest and various maneuvers. Acta Physiol Scand 92:303-317.

Hallin RG, Torebjörk HE (1974b) Methods to differentiate electrically induced afferent and sympathetic $\mathrm{C}$ unit responses in human cutaneous nerves. Acta Physiol Scand 92:318-331. 
Hammarberg HB, Forster C, Torebjörk E (2002) Parameter estimation of human nerve $\mathrm{C}$-fibers using matched filtering and multiple hypothesis tracking. IEEE Trans Biomed Eng 49:329-336.

Hodgkin AL, Huxley AF (1952) A quantitative description of membrane current and its application to conduction and excitation in nerve. J Physiol (Lond) 117:500-544.

Jorum E, Lundberg LE, Torebjork HE (1989) Peripheral projections of nociceptive unmyelinated axons in the human peroneal nerve. J Physiol (Lond) 416:291-301.

Kiernan MC, Mogyoros I, Hales JP, Gracies JM, Burke D (1997) Excitability changes in human cutaneous afferents induced by prolonged repetitive axonal activity. J Physiol (Lond) 500:255-264.

Kocsis JD, Swadlow HA, Waxman SG, Brill MH (1979) Variation in conduction velocity during the relative refractory and supernormal periods: a mechanism for impulse entrainment in central axons. Exp Neurol 65:230-236.

Kocsis JD, Malenka RC, Waxman SG (1983) Effects of extracellular potassium concentration on the excitability of the parallel fibres of the rat cerebellum. J Physiol (Lond) 334:225-244.

Lucas K (1917) The conduction of the nervous impulse. Monographs on physiology. London: Longmans, Green.

Rang HP, Ritchie JM (1968) On the electrogenic sodium pump in mammalian non-myelinated nerve fibres and its activation by various external cations. J Physiol (Lond) 196:183-221.

Raymond SA (1979) Effects of nerve impulses on threshold of frog sciatic nerve fibres. J Physiol Lond 290:273-303.

Raymond SA, Lettvin JY (1978) After-effects of activity in peripheral axons as a clue to nervous coding. In: Physiology and pathobiology of axons (Waxman SG, ed), pp 203-225. New York: Raven.

Raymond SA, Thalhammer JG, Popitz BF, Strichartz GR (1990) Changes in axonal impulse conduction correlate with sensory modality in primary afferent fibers in the rat. Brain Res 526:318-321.

Schmelz M, Schmidt R, Handwerker HO, Torebjork HE (2000) Encoding of burning pain from capsaicin-treated human skin in two categories of unmyelinated nerve fibres. Brain 123:560-571.

Schmidt R, Schmelz M, Forster C, Ringkamp M, Torebjörk HE, Handwerker H (1995) Novel classes of responsive and unresponsive C nociceptors in human skin. J Neurosci 15:333-341.

Schmidt R, Schmelz M, Bickel A, Weidner C, Torebjörk HE, Hand- werker HO (1998) Innervation territories of mechanoinsensitive C nociceptors in human skin. Soc Neurosci Abstr 24:383.

Serra J, Campero M, Ochoa J, Bostock H (1999) Activity-dependent slowing of conduction differentiates functional subtypes of $\mathrm{C}$ fibres innervating human skin. J Physiol (Lond) 515:799-811.

Shin HC, Raymond SA (1991) Excitability changes in C fibers of rat sciatic nerve following impulse activity. Neurosci Lett 129:242-246.

Shin HC, Lee YL, Kwon HY, Park HJ, Raymond SA (1994) Activitydependent variations in conduction velocity of $\mathrm{C}$ fibers of rat sciatic nerve. Neurosci Res 19:427-431.

Stys PK, Ashby P (1990) An automated technique for measuring the recovery cycle of human nerves. Muscle Nerve 13:750-758.

Swadlow HA, Waxman SG (1976) Variations in conduction velocity and excitability following single and multiple impulses of visual callosal axons in the rabbit. Exp Neurol 53:128-150.

Thalhammer JG, Raymond SA, Popitz BF, Strichartz GR (1994) Modality-dependent modulation of conduction by impulse activity in functionally characterized single cutaneous afferents in the rat. Somatosens Mot Res 11:243-257.

Torebjörk HE (1974) Afferent C units responding to mechanical, thermal and chemical stimuli in human non-glabrous skin. Acta Physiol Scand 92:374-390.

Torebjörk HE, Hallin RG (1974) Identification of afferent C units in intact human skin nerves. Brain Res 67:387-403.

Torebjörk HE, Schady W, Ochoa JL (1984) A new method for demonstration of central effects of analgesic agents in man. J Neurol Neurosurg Psychiatry 47:862-869.

Weidner C, Schmelz M, Schmidt R, Hansson B, Handwerker H, Torebjork E (1999) Functional attributes discriminating mechanoinsensitive and mechano-responsive $\mathrm{C}$ nociceptors in human skin. J Neurosci 19:10184-10190.

Weidner C, Schmidt R, Schmelz M, Hilliges M, Handwerker HO, Torebjörk HE (2000a) Time course of post-excitatory effects separates afferent human C fibre classes. J Physiol 527:185-191.

Weidner C, Schmidt R, Hilliges M, Ørstavik K, Schmelz M, Torebjörk HE, Handwerker HO (2000b) Contrast enhancement in peripheral $\mathrm{C}$-fibers is more pronounced in mechano-insensitive than in $\mathrm{CMH}$ fibers. Soc Neurosci Abstr 26:180. 